\title{
Reviewing Manuscripts for Scientific Journals
}

\author{
Robert L. Balster
}

\section{Introduction}

One of the main moral principles of virtually all major religions and cultures is the ethic of reciprocity, sometimes known as the Golden Rule: Treat others as you would like to be treated. Show mutual respect. This Golden Rule is also a fundamental principle of the process of peer review, including the review of manuscripts submitted to professional journals. If you have been asked to review someone's article, it is very likely the case that you are an author yourself and will have been subjected to the same peer-review process. Keeping in mind how you expect your own journal submissions should be reviewed, you could readily derive from that experience nearly all of the advice I will be offering you in your role as a reviewer.

\section{Goal}

The goal of this chapter is to provide rather specific principles and suggestions on how to be a competent reviewer. Most editors of peer-reviewed journals see the peer-review process similarly, even if some of their specific journal policies differ. It is those commonalities that I will address here, approaching the topic from my various roles as former editor-in-chief of Drug and Alcohol Dependence, member of several editorial boards for other journals, and reviewer for

\section{How to cite this book chapter:}

Balster, R L. 2017. Reviewing Manuscripts for Scientific Journals. In: Babor, T F, Stenius, K, Pates, R, Miovský, M, O’Reilly, J and Candon, P. (eds.) Publishing Addiction Science: A Guide for the Perplexed, Pp. 245-263. London: Ubiquity Press. DOI: https://doi.org/10.5334/bbd.m. License: CC-BY 4.0. 
many others. Interested readers may also want to consult prior publications on peer review in general (Moghissi, Love \& Straja, 2013; Godlee \& Jefferson, 2003) and on peer review of journal submissions specifically (Girden \& Kabacoff, 2010; Smart, Maisonneuve \& Polderman, 2013; Hames, 2007). Chapters 7, 8, 9, and 12 in this book also provide information that is relevant to journal reviewing.

I should mention that reviewers of journal submissions are sometimes referred to as referees. I am not aware of any distinction between being a reviewer and a referee. I have always preferred the term reviewer because referee conjures images of a sporting contest with winners and losers. When you are a good reviewer, everyone wins-authors, editors, and the scientific community-because reviewing is fundamentally a constructive process. Yes, reviewing involves making judgments and recommendations, but reviewers are not the decision makers in the process. This responsibility falls to the editor. Thus, I will use the words reviewing and reviewer throughout but just as a matter of personal preference. It seems likely that the words used for the reviewer function in non-English languages have nuances of their own to consider, but that is a matter for another discourse by someone with greater language skills than I.

\section{Brief Overview of the Journal Review Process}

If a journal declares itself to be a "peer-reviewed" journal, this normally means that all articles that are eventually published in that journal have been reviewed. Peer review implies review by outside reviewers as well as the editorial staff. Of course, journals differ in their application of the peer-review process, and it is common for journals to publish editorials, commentaries, book reviews, and similar content without peer review, reserving that form of assessment for research reports and critical reviews.

Before moving on to a more detailed discussion of journal reviewing, I want to give a brief overview of the process so readers can appreciate the steps involved. Box 13.1 outlines the basic steps of the review process used by most journals, keeping in mind that editorial structure differs among journals. For most journals today, submissions are made using an interactive Internet-based system, whereby all articles are processed in a centralized editorial office, or at least as email attachments. The editor then decides whether to edit the article or assign it to some kind of associate or assistant editor. Some journals may have more than one submission office or site, depending on where the author comes from or the general topic of the article. Some journals may have more than one editor look at the submission before assigning it to reviewers, whereas others may have an editorial team that considers the recommendations of the reviewers. To simplify this discussion, I will assume there is one "decision editor" who assigns reviewers and makes decisions. I will refer to this individual as the editor, regardless of the specific editorial title assigned by the journal. 
1. Editor develop a reviewer database.

2. Authors submit manuscripts to the journal.

3. Editor(s) make an initial assessment to decide if the article is suitable for the journal and if peer review is warranted.

4. Editor selects reviewers and invites them to review.

5. Editor monitors the timeliness of peer review and sends reminders or invites new reviewers if necessary.

6. Reviewers complete the review and provide recommendations and comments to the editor and comments to the authors.

7. Editor makes decision to accept the submission, asks authors for a revision, or rejects the submission.

8. Most journals notify reviewers of the editor's decision.

9. If required, authors revise submissions and return to the editor.

10. Editor decides if further review is needed; if so, the process recommences at Step 4.

Box 13.1: Steps in the Journal Review Process.

The first step in the peer-review process is the assignment of reviewers. In the next section, I will discuss reviewer databases and how editors select and invite reviewers (Steps 1 and 4). All journals have a procedure for monitoring the review process after reviewers have been assigned (Step 5). This has become easier with computer-based systems that notify the editorial team when assigned reviewers decline the invitation to review, when reviews are completed, and when they are late.

At some point, the editor stops the review process and decides whether to accept the manuscript for publication (Steps 6 and 7). Editors carefully consider the recommendations of the reviewers and their comments on the article, but ultimately editors themselves must make the decision based on both the reviews and their own assessment of the submission. Editors usually inform reviewers of the decision (Step 8), and often share the comments of all the reviewers with each other, which I believe is a good practice to strengthen the review process.

There are three basic options open to the editor. First, the editor may accept the submission for publication as submitted. For many journals, it is rare to accept a first submission of an article without asking for any revisions at all, but it does happen.

Second, if the submission seems to the editor to be potentially publishable, he or she will ask the authors to make revisions (Step 9). Most journals divide revisions into minor and major categories. Minor revisions do not change the article very much, and the resubmission usually does not require additional 
outside reviews. Major revisions typically require significant changes to the article, such as the collection of additional data, a change in the way the existing data are analyzed or presented, or even changes to some of the conclusions of the report. Manuscripts with major revisions are often returned to the same peer reviewers for their comments and recommendations, although at times an editor may send the manuscript to different reviewers (Step 10).

The third option open to editors is rejection of the submission. Some submissions may be rejected without peer review (Step 3), such as when the topic is not appropriate for the journal readership or the form of article (e.g., case report, book review) is not one published by the journal. Editors may also decide that the article's methods or other characteristics give it little or no chance of receiving a positive recommendation in the peer-review process. Giving immediate feedback on such articles may be in the authors' best interest, because it allows them to submit elsewhere without delay. Rejection without review also saves the time of busy reviewers, who are more useful to the journal when reviewing manuscripts with a greater chance of success. Most often, editors base their rejections on negative recommendations in the peer-review process. In such cases, authors usually receive comments from the editor or reviewers that describe some of the weaknesses of their submission. Ideally, authors consider these comments and revise the article before they send it to another journal.

One of the newer developments in journal publishing is the cascading of journal submissions within publishing consortia of individual journals (Barroga, 2013). With the author's permission, editors can forward a rejected submission and the completed reviews of an article to another journal within the consortium where the article may have a better chance of final acceptance. This saves reviewer time and can expedite final publication because the review process does not need to start all over again. A typical consortium includes journals within a publishing company.

\section{How Do Editors Select Reviewers?}

As we turn to the principle of reciprocity, who do we, as authors, want assigned as reviewers of our article? To be honest, we probably prefer reviewers who are known to be favorably impressed with our work. But minimally, we want reviewers who are knowledgeable about our field of study and who will be fair in the review process. This is what editors want too; they want competent reviewers with specialized knowledge of the potential advantages and pitfalls of various research approaches. They also want reviewers who are fair and unbiased, without conflicts of interest. Finally, editors want reviewers who complete their work on time and who write constructive comments about the submission.

The process of selecting appropriate reviewers usually involves the use of a database. At a minimum, such a database includes email addresses by which to contact reviewersIn the case of a large, multidisciplinary journal, editors also 
need some means of matching reviewers with submissions. Smaller, more specialized journals often rely mainly on their normal editorial advisory boards for reviews, but ad hoc reviewers carry most of the load for larger journals.

In addition to up-to-date contact information, a typical reviewer database includes some means of identifying their reviewers' areas of expertise. For example, the database may assign to each reviewer one or more keywords or classifications (e.g., molecular genetics, pharmacotherapy, prevention, policy). Commercial editorial software systems are particularly adept at matching the keywords assigned to both submissions and reviewers to provide editors with a list of knowledgeable reviewers. These programs also tell editors if reviewers are currently assigned other manuscripts, the date of their last review, and the number of reviews they have done lately. It is also possible to see if reviewers have defaulted on prior assignments or how long they have taken to complete their prior reviews. Some systems even allow editors to rate reviewers to help them remember who provided useful suggestions and constructive and timely comments in previous reviews.

There are several ways to get one's name added to a journal's database. Perhaps the most common way is by publishing an article in that journal. Editors typically prefer reviewers who themselves have published several articles as senior or corresponding author. If a suitable reviewer is not already in the database, many editors do a quick author search using PubMed or other search tool to see if potential reviewers have other publications, and the editorial software often facilitates this search. Sometimes authors suggest reviewers for their manuscripts. If the editor agrees with the suggestion and the reviewer is not in the database, the reviewer will be added. Editors may ask their editorial advisory boards to suggest new reviewers for the database, and most editors are also pleased to receive self-nominations. Finally, an editor sometimes receives submissions for which no suitable reviewer is available. In such cases, editors use their knowledge of the field or use authors cited in the submission who are clearly doing related work to add new reviewers to the database.

Selecting the best possible reviewer for a submission is one of the most important responsibilities of an editor. In my experience, taking time at this step to ensure a good match between reviewer and submission will often save timeand requests for more reviews-later on. Reviewers, too, prefer to assess articles in areas in which they feel qualified. Each editor goes about the matching process in a different way. In addition to seeking reviewers with expertise in the area, editors also may seek to balance their selection of reviewers for a single submission along several dimensions. For example, editors may seek a methodological balance, in which a reviewer with specific knowledge of a data-analytic approach complements another with knowledge of the content area of the work. It is often good to pair a senior scientist with a less-experienced reviewer because this can serve to train the junior person who subsequently sees the comments of the senior reviewer. Sometimes the editor needs a reviewer known to be unbiased in a particularly controversial area. Editors usually like to have reviewers 
who provide a broad perspective on the work to ensure geographical, cultural, or gender balance.

In general, only two reviewers are needed for each submission, but there are times when more than two are invited. If the review process becomes delayed because of problems obtaining timely reviews, editors may add a reviewer they know to be particularly reliable. These and many other subtle factors make the reviewer-selection process a challenging one.

In addition to selecting knowledgeable and reliable reviewers, editors do their best to avoid actual or perceived conflicts of interest (COIs) and bias. As I will discuss later, editors cannot know about every possible COI or bias that reviewers may have; therefore, they rely on reviewers to tell them. There are some relatively straightforward methods editors use to try to avoid apparent COIs. They generally exclude as potential reviewers scientific colleagues close to the author, such as reviewers who have co-authored in recent years with any of the authors of the submission or persons known to be part of the same research team. It is usually wise not to assign reviewers from the same institution as the authors, but, in the case of authors based at large, multicampus institutions, such precautions may not always be necessary. Because editors are usually experts themselves, they may be aware of longstanding disagreements or controversies and take care to select unbiased reviewers. It is impossible to eliminate potential bias completely, but editors do the best they can.

The actual process of inviting reviewers differs among journals. Some editors send a copy of the submission and reviewing instructions directly to the reviewer. Other editors first invite possible reviewers, usually by sending the abstract by email and asking them first to agree to do the review by a certain deadline. If the reviewers agree, they are then assigned the review and provided with access to the full article, review forms, and instructions. One advantage to this invitation process is that potential reviewers can identify COIs they may have or indicate that they lack expertise in the area of study. Some editors will invite several reviewers and then assign only the first two who agree. Computer technology and the use of email have automated some of these steps, making the process faster. It is now possible to receive a submission, invite reviewers, assign them, and get the review process started all in one day.

\section{Why Have Peer Review?}

Before further discussion of how to be a good reviewer, I will explain why we use peer review and why someone would want to be a peer reviewer. Peer review has four primary objectives: (a) advise the editorial decision-making process, (b) justify rejections, (c) improve the quality of acceptable manuscripts, and

(d) identify instances of ethical or scientific misconduct. 


\section{Editorial Decision Making}

The most obvious reason to seek reviews of journal submissions is to help the editor make a decision about the article's acceptability for publication in that journal. One should always remember that reviewers only make recommendations; it is the editor who chooses whether to follow those recommendations. This fact often frustrates reviewers, who may feel that the editor ignored their advice. A consideration of some of the factors that affect the editor's decisions can relieve some of that frustration. Editors must make decisions based on all of their reviewers' input, and sometimes different reviewers give conflicting recommendations. The editor may feel that a problem identified by a reviewer could be addressed in a revision or that the author may have good arguments for why the problem is not an important consideration. Indeed, the editor may read the article and disagree with the reviewer's interpretation. An editor may feel that the importance of the article mitigates some problems identified by the reviewers. On the other hand, reviewers may not find any fault with an article that the editor decides carries little impact or belongs in a different journal. Most journals receive more submissions than they can publish and thus editors must choose among potentially publishable articles with no major flaws. All of these factors and others go into the editorial decision-making process. Editors who choose not to follow a reviewer's recommendations may still consider the review excellent. Reviewers who are provided access to the editor's decision letter, or to the comments of the other reviewer, can usually glean the basis for the editor's decision.

\section{Explaining the Basis for Rejection}

Nearly all journals ask reviewers to provide comments and suggestions on the submission, which are then sent to the author with the decision letter. When reviewers recommend rejection of a manuscript, they should provide the basis for that recommendation in their comments to the authors. Not only does this give authors the feedback they deserve on their submissions, but it also helps the authors to revise their articles for submission elsewhere and to improve their work in general.

\section{Improving the Quality of Published Articles}

Certainly, one of the most important reasons for obtaining constructive comments during the review process is to make the articles that are ultimately published the best they can possibly be. I have seen modest articles transformed during the review process into far better and more important contributions to the field. I received many statements of appreciation by authors for the 
improvements in their manuscripts brought about by peer review. Of course, not all authors appreciate constructive criticism or relish doing the extra work required to revise their submissions to satisfy reviewers, but I believe that most authors value expert criticism of their work. I suppose that some contributions could be weakened when authors follow reviewer advice, but I cannot recall a specific example where I know this occurred. It is the responsibility of the editor to make judgments about major changes reviewers ask authors to make and to tell authors in a cover letter if the editor does not agree with a reviewer's suggestions. Editors should also carefully consider counter arguments made by authors who prefer not to make some recommended changes.

What is the scientific evidence that peer review improves the quality of scientific publication? Although this chapter is not the place to have a thorough discussion of this topic, it may interest the reader to know that there has been relatively little research on this topic, and the work that has been done does not provide a clear answer to the question (Fletcher \& Fletcher, 2003; Overbeake \& Wagner, 2003; Jefferson et al., 2007). Nor is there much empirical research on various approaches to peer review (e.g., open vs. blinded review). Criticisms have been published of the peer-review process for both journal publications and research grant applications (e.g., Smith, 2006). Nonetheless, editors in general see articles improve through the peer-review process, but perhaps they have a bias, and peer review is the only system we have for quality control in journal publication.

\section{Identify Areas of Ethical or Scientific Misconduct}

Most journals request that reviewers comment on any research subjectprotection issues or other ethical concerns they detect in a submission. Reviewers' careful examination of the data may reveal inconsistencies between reported methods and the ways in which data are presented or analyzed or may uncover highly unlikely data sets (e.g., with no variability) that may lead reviewers to suspect errors in data reporting or outright data fabrication.

Reviewers may know of concurrent submissions by the same authors of the same manuscript with the same data to two or more journals or prior publications. It is important for reviewers to communicate their concerns about possible ethical or scientific-misconduct problems to the editor. Typically, this is done in confidential comments to the editor, who then must investigate these concerns. Reviewers do not need proof of these types of problems, just a reasonable basis for concern.

\section{Why be a Journal Reviewer?}

In the following, I expand on seven of the main reasons researchers review manuscripts for scientific journals. But keep in mind that the most important 
reason many people do it is this: They enjoy it. Nonetheless, thinking critically about science, staying informed of the latest advances, and making a contribution to health are what attracted many of us to science in the first place. Reviewing is a scholarly, creative, focused, important activity that is capable of being completed with a few hours of work. What could be better?

\section{Fulfill Your Professional Responsibility}

The peer-review process is a simple application of the Golden Rule. You need people to review your articles, and therefore you should review those of others. A system of all authors and no reviewers is doomed. Thus, it is your professional responsibility to be part of the process, both as an author and reviewer. I do not subscribe to the view that senior scientists can be excused from the peer-review process because they "did their duty" earlier in their careers. If you write, you should review. Indeed, the perspective of experienced scholars can be particularly important.

\section{Improve Your Understanding of the Peer-Review Process}

Younger scholars particularly need to learn the peer-review process, because much of their career success will depend on it. Many scientific mentors include in their training a gradual exposure to doing peer reviews. A good strategy is for a mentor to ask a junior colleague to prepare a review of a manuscript that has been assigned to the mentor. The mentor should explain fully the confidentiality issues surrounding the review process when asking a junior colleague for help. The novice reviewer then returns the review to the mentor who modifies it as needed and gives feedback to the trainee. The mentor then submits the review to the journal. In such instances of guided peer review, mentors should tell the editor the name of the junior colleague who helped with the review. Mentors might even recommend that the junior colleague be added to the reviewer database once they feel the colleague is ready to do independent work.

\section{Improve Your Critical Thinking}

The review of other people's work improves your critical thinking about your own work. Good reviewers attempt to articulate both the strengths and weaknesses of a particular scientific approach. You may be using a similar approach in your own work without thinking about it critically as often as you should. Perhaps you are considering the use of methods similar to those in the article you are reviewing. Thinking about some of the article's weaknesses can lead you to improve the approach. 
One of the most useful ways to evaluate your own critical thinking is to see what the other reviewer(s) and editors say about the work. I strongly believe that journals should make all comments to authors and decision letters available to reviewers on an anonymous basis, although not all journals do. When another reviewer or the editor identifies a serious flaw in experimental design or data analysis that you missed, it can be both instructive and a little embarrassing.

\section{Improve Your Own Writing and Data Presentation}

Reviewing a manuscript (and comments from other reviewers and the editor) gives you new insights into how to improve your own writing, presentation, and data analyses. By seeing how authors make revisions and respond to reviewer comments, you can improve your own approach to revising articles. Reviewers often advise authors on how articles can be shortened and more sharply focused. As reviewers help authors focus their writing, they likewise learn to do this with their own articles. In addition, proofreading other manuscripts for errors improves the proofreading of your own writing.

\section{Learn More about Research in the Field}

Reviewing a scientific article can give a reviewer a much better understanding of the work than does just reading it because of the critical thinking involved in doing a review. In addition, you will probably be asked to review articles that you would not normally read because they are a little outside your specific area of work. The comments and suggestions of the editor and other reviewer(s) are sometimes more interesting and important to you than the article you reviewed. Some of the best scientific writing I have encountered has taken the form of reviews. After all, reviewers are experts in the field who are asked to summarize the salient strengths and weaknesses of a scientific study or new hypothesis in a few paragraphs.

\section{Build Relationships with Journals}

The databases that include you as a reviewer generally will also include you as an author. Persons who are regular authors and reviewers for a journal and who are successful in both these roles build a relationship with a journal, becoming good "journal citizens." The editorial team comes to know who you are, appreciate better your areas of expertise, and develop confidence in your work. Your good relationship with a journal may result in your being asked to join its editorial advisory board or take on editing roles yourself. 


\section{Fulfill Service Obligations for Promotion}

As scientists, our work is constantly being evaluated. This includes assessment for promotion and/or tenure. For most scholars, service to the profession is one of the areas where we are evaluated and judged. As mentioned above, journal reviewing is an important service that should be acknowledged and rewarded. I know that reviewers typically report their journal reviewing activities on their curricula vitae and include them in regular activity reports. Simply being invited to review a manuscript is evidence that you are known in the field and that an editor has some confidence in your expertise. Many journals regularly publish lists of recent reviewers and some use additional incentives (such as small gifts or reduced costs for the publisher's books and journals) to reward good reviewers.

\section{How to be a Good Reviewer}

Much of the advice in this section stems from the goals of the review process, as described above. Simply stated, a good reviewer helps the editor achieve the goals of peer review. Another guiding principle is our friend the Golden Rule. You should be the kind of reviewer that you would want to review your work. It is always important for reviewers to try to take an author's perspective and to remember that publishing is vitally important to authors. Sometimes an author needs one more publication to ensure a promotion or to receive a favorable review on a grant application. Authors may be performing research in a highly competitive area where having an article accepted for publication is crucial evidence of their precedence. Reviewers should easily be able to imagine an author's response to a careless review or one that is delayed by months. Reviewers often offer excuses to the editor for late or cursory reviews, but the author feels the delay-and the curt treatment-even more keenly than the editor. I suggest placing the following aphorism on your desk as you participate in the review process: Review others as you would like to be reviewed.

Below, I present what could be viewed as one former editor's advice on being a good reviewer. I have ordered this section essentially in the order of steps in the review process as presented in Box 13.1.

\section{Respond Promptly to Invitations to Review}

As I mentioned above, many journals now use email or fax to invite reviewers for a submission. The invitation typically includes only basic information about the article and an abstract. The worst thing you can do with an invitation is ignore it. It takes only a minute or two to decide if you are able to accept the 
invitation and then notify the editor. If you want to do the review but cannot complete it by the deadline, contact the editor and see if a later deadline is acceptable. If you decide you cannot accept the invitation, you can help the editor with some suggestions for other reviewers, but the main thing is to tell the editor promptly. Failure to reply puts the editor in the difficult situation of deciding how long to wait until contacting other potential reviewers and puts the review process behind schedule. To avoid delays with this invitation step, some editors invite several qualified reviewers and hope that the required number will agree promptly.

Reviewers decline invitations all the time. Editors are used to this. If you are too busy at the moment, have other review assignments to complete, do not feel competent to review the article, or have a COI, editors will understand, especially if you regularly agree to write reviews for that journal. If you will never agree to do a review for that journal, it is best to tell the editor so you can be removed from the reviewer database.

\section{Notify Editor of Any Potential COIs or Previous Reviews You Did of the Article}

If you have an obvious COI, you should decline the invitation to review. Often reviewers are uncertain if they should declare what appears to be a slight COI, such as a small collaboration with an author or a collaboration that occurred many years ago. In these cases, it is best to tell the editor about your concerns, who can then decide if it represents a real conflict. Putting such ambiguous conflicts on the record can often lessen an editor's concern. On the other hand, if you feel there might be a conflict that might affect your ability to give a fair and unbiased assessment, then you probably are in conflict and should not accept the invitation to be a reviewer.

More often than one might expect, reviewers are invited to review articles they have already reviewed and rejected for other journals. Many reviewers are in more than one reviewer database, and these journals match reviewers to submissions in much the same way. Some reviewers prefer to decline the second invitation, often stating that they do not want to place the author in double jeopardy. In such instances, I recommend asking the editor what to do. I personally had no problem with reviewers assessing the same manuscript for two different journals. If the new article is identical to the one reviewed earlier and the reviewer feels that the same recommendation and comments are in order, then he or she should submit them again. But if the author has made improvements before resubmitting to the new journal, the recommendation should address this improved manuscript. Authors should be advised that they take a risk if they submit a rejected article to another journal without addressing the concerns raised with the initial submission, because their manuscript may be assigned to the same reviewer. 


\section{Maintain Confidentiality}

Submissions to journals are confidential information. Reviewers should scrupulously respect the secrecy of the information, including even the existence of the submission. Reviewers who solicit input on a review from a colleague or trainee must first inform the colleague or trainee about the confidentiality of the information. The reviewer is responsible for any disclosures by his or her consultants.

The most unethical use of information in manuscripts under review occurs when the reviewer uses the information to facilitate directly the reviewer's own work, for example by using a new methodology before it has been published or by citing the work in his or her own publication or grant application. Failure to maintain confidentiality and misuse of information obtained in the review process is scientific misconduct.

I strongly advise reviewers not to contact author(s) with questions or offers to negotiate some changes in the manuscript or for any other reasons related to the submission. This applies even if the journal uses an unblinded review process, whereby the authors know the identities of their reviewers. Such communications usually go badly for both reviewer and author, who can end up arguing about the article, and they improperly exclude the editor from the decision-making process, perhaps concealing from him or her important aspects of the review process. It also can damage important scientific relationships among the parties involved.

\section{Complete the Review on Time}

Editors give reviewers a fixed period within which to return a review recommendation. Reviewers who are late ultimately disrespect the author. When a reviewer accepts an invitation to review with a specified deadline, there is little excuse for tardiness. If you know you are going to be late with a review, notify the editor, who can decide whether to wait for your review or invite someone else. One of the most disagreeable aspects of being a journal editor is the need to remind reviewers, often several times, of late reviews. Computerized reviewer databases keep track of how long it takes reviewers to complete reviews so that chronically late reviewers can be removed from the database.

\section{Make a Publication Recommendation}

Completing a review requires at least two steps: making a publication recommendation and writing comments to the authors (and editor, when needed). Both are important, but I begin with some advice about making recommendations. Read the reviewer instructions carefully to learn on what basis the 
journal wants you to make a recommendation. How does the editor want you to balance technical merit versus importance to the field, etc.? Most journals ask for your overall recommendation based on the review criteria specified. Although journals differ on this, they usually want one of four possible decisions: accept, minor revision, major revision, or rejection. Because many journals receive more technically acceptable articles than they can publish, editors want recommendations that also consider the importance of the information and whether it covers new ground or applies a novel perspective. In my experience, reviewers are least comfortable with judging the importance of submissions, but as experts in the area, they may be in the best position to make this judgment.

\section{Provide Confidential Comments to the Editor}

Nearly all journals give reviewers the option of providing comments to the editor. These comments are confidential and not shared with the author or other reviewer(s). There is no need to reproduce your comments to the author, but do include things you believe the editor should know besides your comments to the author. The following are some of the matters you would want to bring to the confidential attention of the editor.

Identify COIs not previously reported. If you have some relationship to the authors or have some financial or personal interest in the work you are reviewing, you should tell the editor. The editor will take this information into account when making decisions based on your recommendations. If the editor believes the COI precludes you from being a reviewer, your review may not be considered in making a decision and your comments to authors may not be sent on. Situations like this are rare, but it is better to tell the editor too much than too little.

Identify your areas of expertise. There may be some aspects of the article about which you do not feel competent to provide a review. As I mentioned before, you may have been invited to complement another reviewer who lacks expertise in your area. Tell the editor if there are parts of the article for which another expert is needed. It is far better to place statements about your areas of expertise in the comments to the editor than in your comments to the author.

List concerns about ethics or scientific misconduct. As I have already mentioned, you need not be certain of ethical problems to report your suspicion. If you have sufficient cause for question, tell the editor.

Provide other comments. Reviewers may have additional information or some options the editor should consider that are not appropriate to send to the authors. Reviewers should feel free to help editors in any way they can to make the right decision and seek changes in a submission. 
Examples include when a reviewer is familiar with a controversy surrounding the reported research or when there is excessive overlap with other reports coming from the same research group.

\section{Complete Questionnaires}

Most journals ask reviewers to complete questionnaires, which may include items about COIs, ethics, technical merit, significance, language usage, or other matters. Some of these items (e.g., COI and research subject protection) may be crucial to the review process. It should not take you long to complete the questionnaire, and the information you provide will help the editor.

\section{Provide Comments for Authors}

Here again, the Golden Rule tells us how to act. As an author, you would undoubtedly want to know the basis for an editor's decision; so, too, do the authors of the articles you review. Comments to authors are a crucially important part of the review process and should be written for the edification of both the author and the editor. There is no standard length for these comments. In my experience, one to two pages is usually sufficient, but if the key basis for your recommendation can be stated in a paragraph or two, that is fine. Some reviewers do fairly detailed page-by-page suggestions for improvements; these are much appreciated by editors if they are constructive. Below are guidelines for writing a good review.

State the article's main strengths and weaknesses. Start the comments to authors with your views of the main strengths and weaknesses of the manuscript. In general, after reading this section the editor should understand the basis for your final recommendation (which you should not include in the comments to authors). Make your statement of weaknesses as constructive as possible and suggest possible avenues by which the author might address the problems in a revision. Weaknesses that are inherent in the design and execution of the study cannot be fixed in a revision; therefore, you can expend less effort in telling authors how they should have done their study. Even if you recommend rejection, you should provide constructive suggestions for improving the article in the event that the editor gives the author an opportunity to address the weaknesses. Many reviewers organize the comments to authors by describing the strengths and weaknesses of each of the sections of the article (e.g., introduction, methods, results, and discussion).

Try to balance technical merit with scientific significance. This balance has been the subject of debate in the grant-review process for many 
years, with many believing that technically competent but scientifically unimportant or uncreative proposals have an advantage, although they may lack significance. It is important for reviewers of journal publications to identify particularly important or creative articles and, conversely, to indicate if in their judgment an article represents only a minor advance in the field.

Provide specific suggestions for improvements. Detailed suggestions for improving the writing in the article, the figures, or the tables are very helpful to both the editor and the author. Identify paragraphs or sentences that are unclear, point out areas where information is missing, and explain how the writing can be clearer. If you have trouble understanding the article, there is a good possibility that other readers will as well. Reviewers familiar with the journal's formatting requirements can point out departures for correction. Reviewers also can identify relevant publications that should have been cited by the author.

It is important for reviewers to appreciate that journal space is often limited and that articles should be as short as they can be while still covering the necessary material. Reviewers can be especially helpful in pointing out how to shorten articles, perhaps by eliminating tables or figures or summarizing data in text. Introductions are often longer than they need be: Point out nonessential background information that can be removed. Similarly, discussions can be too speculative or focused on minor aspects of the study results. Chapter 12 provides advice on writing articles for addiction journals; reviewers can offer similar advice in their comments to authors. Reviewers are often exceptionally generous with their help, especially to inexperienced authors. I have been truly impressed and am grateful to the many reviewers who see the peerreview process as a mentoring opportunity.

Having advised reviewers to provide both general and specific suggestions for improving manuscripts, I would not want authors to see this as a rationale for submitting rough drafts or articles that have not been proofread so they can get reviewer feedback. Submission of unpolished drafts shows a lack of respect for the reviewers and editors and is not a good application of the Golden Rule. Authors should submit articles that they would be happy to review. Reviewers often become exasperated when reviewing sloppy submissions-it colours their recommendations and discourages them from providing detailed suggestions. Authors should submit their very best work.

Comment on language issues. Chapter 4 discusses the problems faced by authors having to submit their articles to English-language journals when English is not their first language. Reviewers cannot be expected to correct language problems. When assigned an article obviously written by a non-native English speaker, reviewers should do their best to focus on the science being presented and simply point out areas where 
language usage needs to be improved. There are various means by which authors and editors can handle this problem; therefore, reviewers should not be biased in their recommendations based on poor English language usage. Instead of addressing language issues in peer review, some journal publishers provide language assistance, or the editor can request that the author seek help from a native English speaker or a language editing service if the article is likely to be accepted.

Avoid unconstructive comments. Some things do not belong in comments to authors. Foremost are pejorative comments about authors or the work. Reviewers should strive to be constructive at all times. Editors may remove overly personal criticisms or other material that insults the authors, their institution, or their geographical location.

Jokes and witty remarks also do not belong in comments to authors. Editors may appreciate them in comments to the editor, but for authors this is serious business. They may perceive such remarks as a lack of serious intent by the reviewer.

Unless the journal uses unblinded reviews, reviewers should not reveal their identity in the comments to authors. Personal pronouns can provide clues to the reviewer's identity ("We did a study that showed. .."). Often, reviewers provide an author with several of the reviewers' own publications that they feel should have been cited, leading the author to suspect who the reviewers are.

\section{Reviewing Revisions}

Most of what I have said about reviewing applies to reviewing revisions of manuscripts. I feel that reviewers have a special obligation to agree to review revised articles that they reviewed in an earlier version. Reviewers should evaluate how the authors addressed the weaknesses they identified in the earlier version. If authors have failed to address the concerns successfully, it should be stated in this subsequent review. On the other hand, if the authors were successful in their revisions or, alternately, convinced you that your concerns were unwarranted, this should also be stated in your comments. In any case, you should check to make sure the authors actually made the changes in the manuscript. Addressing problems only in a cover letter is not constructive.

It is also helpful for reviewers to look at the concerns of the other reviewer(s) and see how the authors addressed them. If you disagree with the other reviewer(s) and agree with the authors' explanation and defense of their original article, help out the editor by discussing these issues. If you feel that another reviewer was way off base, it is probably best to discuss your concerns in the comments to editor rather than sharing your views with the authors.

Sometimes when authors clarify their methods or data analyses, you see that the submission is even weaker than you initially thought. If that is the case, 
state your new observations clearly, and make your recommendation accordingly. Sometimes in reading a revision of an article, reviewers will identify weaknesses they missed in the first review. It is fine to voice these new concerns in your written review, but ideally you should have identified these problems in your earlier review. Depending on the scope of additional changes you and the editor request in a second revision, the editor may return the same article to you a third time for your recommendation.

\section{What Do Editors Do with Peer Reviews?}

After reviews of a manuscript are completed, the editor evaluates the article and the reviews and makes a decision. As I mentioned, with some journals this decision-making process may involve multiple editors and/or members of the editorial advisory board; others may involve one editor's decision. Many factors go into making a decision on a submission. The primary factor is the recommendations of the reviewers, but other factors include the editor's assessment of the importance of the article and how well it fits with the journal's area of coverage, additional concerns the editor may have that were not identified by the reviewers, the likelihood that the submission can be successfully revised to address its weaknesses, and the overall rejection rate for the journal.

Addiction journals differ greatly in their rejection rates with some able to publish much less than half of the submitted articles. Journal editors far prefer the opportunity to select only the best articles for their journal over not having enough acceptable submissions to fulfill their page budget. Journals with high rejection rates usually have higher impact factors because they choose only top-quality articles, and better and more important articles tend to be cited heavily. It is important for reviewers to understand this dynamic to appreciate why their reviews are so important to both the authors and the journals.

\section{Summary and Conclusions}

I have tried to provide background and helpful advice on reviewing articles for scientific journals. I provided an overview of the peer-review process, reasons for having peer review, why scholars would want to be peer reviewers, and tips on how to be a good reviewer. I have done this primarily from the point of view of a former journal editor but have emphasized authors' points of view as well. I encouraged you to apply the Golden Rule and be the type of reviewer you would want to review your submissions to journals. If you take an author's point of view, it will greatly help you be a good reviewer. Peer review is a very important part of the scientific process. Your work as reviewers is greatly appreciated and necessary. I hope this chapter helps you improve your reviewing skills. 
Please visit the website of the International Society of Addiction Journal Editors (ISAJE) at www.isaje.net to access supplementary materials related to this chapter. Materials include additional reading, exercises, examples, PowerPoint presentations, videos, and e-learning lessons.

\section{References and Further Reading}

Barroga, E. F. (2013). Cascading peer review for open access publishing. European Science Editing, 39(4), 90-91.

Fletcher, R. H., \& Fletcher, S. W. (2003). The effectiveness of journal peer review. In T. Jefferson \& F. Godlee (Eds.), Peer review in health sciences, 2 nd edition (pp. 62-75). London, England: BMJ Books.

Girden, E. R., \& Kabacoff, R. I. (2010). Evaluating Research Articles, 3rd edition. Thousand Oaks, CA: Sage Publications.

Godlee, F., \& Jefferson, T. (Eds). (2003). Peer review in health sciences, 2cd edition. London: BMJ Books.

Hames, I. (2007). Peer review and manuscript management in scientific journals: Guidelines for good practice. Hoboken NJ: Wiley-Blackwell.

Jefferson, T., Rudin, M., Folse, S. B., \& Davidoff, F. (2007). Editorial peer review for improving the quality of reports in biomedical studies. Cochrane Database of Systematic Reviews. DOI: https://doi.org/10.1002/14651858.MR000016. pub3

Jefferson, T., \& Godlee, F. (Eds.). (2003). Peer review in health scheinces, $2 \mathrm{~cd}$ edition. London: BMJ Books.

Moghissi, A. A., Love, B. R., \& Straja, S. R. (2013). Peer Review and Scientific Assessment. Alexandria, VA: Institute for Regulatory Science.

Overbeke, J., \& Wagner, E. (2003). The state of evidence: What we know and what we don't know about journal peer review. In F. Godlee and T. Jefferson (Eds.), Peer review in health sciences (2nd ed.). London, England: BMJ Books.

Smart, P., Maisonneuve, H., \& Polderman, A. (Eds.). (2013). Science Editor's Handbook, 2cd edition. Cornwall, UK: European Associatoin of Science Editors (especially Section 4).

Smith, R. (2006). Peer review: A flawed process at the heart of science and journals. Journal of the Royal Society of Medicine, 99, 178-82. 
